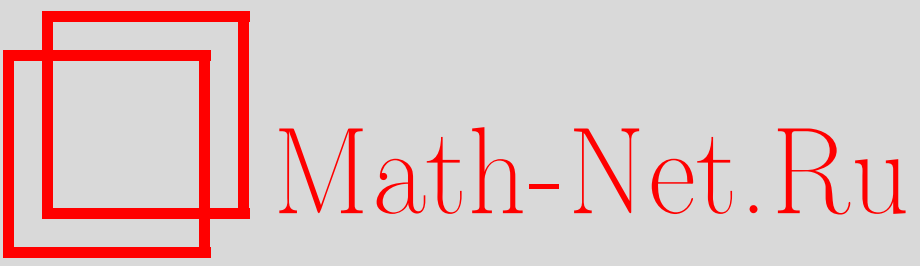

А. В. Гиль, А. И. Задорожный, В. А. Ногин, Обращение и описание некоторых потенциалов с $L^{p}$-плотностями в неэллиптическом случае, Becmн. Сам. гос. техн. ун-та. Сер. Физ.-мат. науки, 2011, выпуск 4(), 43-49

DOI: https://doi.org/10.14498/vsgtu1002

Использование Общероссийского математического портала Math-Net.Ru подразумевает, что вы прочитали и согласны с пользовательским соглашением

http://www.mathnet.ru/rus/agreement

Параметры загрузки:

IP : 107.22 .136 .117

26 апреля 2023 г., 18:29:14 


\title{
Функциональный анализ
}

\author{
УДК 517.983
}

\section{ОБРАЩЕНИЕ И ОПИСАНИЕ НЕКОТОРЫХ ПОТЕНЦИАЛОВ С $L^{P}$-ПЛОТНОСТЯМИ В НЕЭЛЛИПТИЧЕСКОМ СЛУЧАЕ}

\section{А. В. Гиль, А. И. Задорожннй, В. А. Ногин}

Южный федеральный университет,

Факультет механики, математики и компьютерных наук,

344090, Ростов-на-Дону, ул. Мильчакова, 8а.

E-mails: gil-alexey@yandey.ru, simon@rsu.ru, vnogin@math.rsu.ru

Строится обращение обобщенных потенциалов Стрихарца с особенностями ядер на конечном объединении сфер в $\mathbb{R}^{n}$ с плотностями из пространства $L^{p}$, $1 \leqslant p \leqslant 2$ и из пространства Харди $H^{1}$ в неэллиптическом случае, когда их символь вырождаются на множестве меры нуль в $\mathbb{R}^{n}$. Даётся также описание рассматриваемых потенииалов в терминах обращающих конструкиий.

Ключевые слова: свёртка, осциллирующий символ, мультипликатор, обобщённая функиия.

Введение. Рассматривается оператор свёртки

$$
M_{\theta}^{\bar{\beta}} \varphi=m_{\theta}^{\bar{\beta}} * \varphi
$$

с ядром, имеющим степенные особенности на конечном объединении сфер:

$$
\begin{aligned}
m_{\theta}^{\bar{\beta}}(y)=\theta_{1}(|y|)\left(r_{1}^{2}-|y|^{2}+\right. & i 0)^{\beta_{1}-1} \times \ldots \times \theta_{s-1}(|y|) \times \\
& \left.\times r_{s-1}^{2}-|y|^{2}+i 0\right)^{\beta_{s-1}-1} \theta_{s}(|y|)\left(1-|y|^{2}\right)_{+}^{\beta_{s}-1},
\end{aligned}
$$

где $\bar{\beta}=\left(\beta_{1}, \ldots, \beta_{s}\right), \beta_{j}>0,1 \leqslant j \leqslant s, s \geqslant 2,0<r_{1}<r_{2}<\ldots<r_{s-1}<r_{s}=1$. Здесь $\theta_{j}(r)$ - гладкие функции, $\theta_{j}\left(r_{j}\right) \neq 0,1 \leqslant j \leqslant s$.

Операторы вида (1) возникают при решении задачи Коши для волнового уравнения (см. [1-3]). $\left(H^{p}-H^{q}\right)$-оценки для этих операторов, $0<p \leqslant q<\infty$, были получены в [4].

Методом аппроксимативных обратных операторов (АОО) строится обращение потенциалов $(2)$ с плотностями из пространства $L^{p}, 1 \leqslant p \leqslant 2$ и из пространства Харди $H^{1}$ в неэллиптическом случае, когда их символы вырождаются на множестве меры нуль в $\mathbb{R}^{n}$. Даётся также описание образов $M_{\theta}^{\bar{\beta}}\left(L^{p}\right)$ и $M_{\theta}^{\bar{\beta}}\left(H^{1}\right)$ в терминах обращающих конструкций.

Алексей Викторович Гиль (к.ф.-м.н.), ст. преподаватель, каф. дифференциальных и интегральных уравнений. Анатолий Иванович Задорожный (д.ф.-м.н., проф.), зав. кафедрой, каф. дифференциальных и интегральных уравнений. Владимир Александрович Ногин (к.ф.-м.н., доц.), доцент, каф. дифференциальных и интегральных уравнений. 
1. Предварительные сведения. Введём следующие обозначения: $(F f)(\xi)=$ $=\widehat{f}(\xi)=\int_{\mathbb{R}^{n}} f(x) e^{i \xi x} d x$ - преобразование Фурье функции $f ;\left(F^{-1} f\right)(\xi)=$ $=\tilde{f}(\xi)=(2 \pi)^{-n}(F f)(-\xi)$ - обратное преобразование Фурье; $R^{0}\left(\mathbb{R}^{n}\right)=\{f:$ $\left.f(x)=\widehat{\varphi}(x), \varphi(x) \in L^{1}\left(\mathbb{R}^{n}\right)\right\}$ - винеровское кольцо; $\mathcal{S}$ - класс Шварца быстро убывающих гладких функций; $\mathcal{S}^{\prime}$ - пространство обобщённых функций медленного роста; $C_{0}\left(\mathbb{R}^{n}\right)=\left\{f: f \in C\left(\mathbb{R}^{n}\right), f(\infty)=0\right\}$ - пространство непрерывных функций, исчезающих на бесконечности; $W_{\varepsilon} \varphi=w_{\varepsilon} * \varphi$ - интеграл Гаусса-Вейерштрасса, где $w_{\varepsilon}(x)=(4 \pi \varepsilon)^{-n / 2} e^{-|x|^{2} /(4 \varepsilon)}\left(\hat{w}_{\varepsilon}(\xi)=e^{-\varepsilon|\xi|^{2}}\right) ; \Phi, \Psi-$ пространства П. И. Лизоркина: $\Psi=\left\{\psi \in \mathcal{S}:\left(D^{\nu} \psi\right)(0)=0,|\nu|=0,1, \ldots\right\}$, $\Phi=\{\varphi \in \mathcal{S}: \hat{\varphi} \in \Psi\}$.

Через $H^{1}=H^{1}\left(\mathbb{R}^{n}\right)$ обозначим множество всех $\mathcal{S}^{\prime}$-распределений таких, что

$$
f^{+}(x)=\sup _{0<\varepsilon<\infty}\left|\left(f * \varphi_{\varepsilon}\right)(x)\right| \in L^{1},
$$

где $\varphi \in \mathcal{S}$ и $\int_{\mathbb{R}^{n}} \varphi(x) d x \neq 0, \varphi_{\varepsilon}(x)=\varepsilon^{-n} \varphi\left(\frac{x}{\varepsilon}\right)$ и $\left(f * \varphi_{\varepsilon}\right)(x)=\left\langle f, \varphi_{\varepsilon}(x-\cdot)\right\rangle$. Положим $\|f\|_{H^{1}}=\left\|f^{+}\right\|_{L^{1}}$ (см. [5, с. 269]).

Пусть $V$ - произвольное замкнутое множество в $\mathbb{R}^{n}$. Через $\Psi_{V}$ обозначим класс всех функций из $\mathcal{S}$, которые исчезают вместе со всеми своими производными на $V$ :

$$
\Psi_{V}=\left\{\psi(\xi) \in \mathcal{S}: D^{k} \psi(\xi)=0, \xi \in V,|k|=0,1,2, \ldots\right\} .
$$

Через $\Phi_{V}$ обозначим класс прообразов Фурье функций из $\Psi_{V}: \Phi_{V}=$ $=F^{-1}\left(\Psi_{V}\right)$.

Пространства $\Psi_{V}$ и $\Phi_{V}$ были введены и изучены С. Г. Самко (см. $\left.[6, \S 3]\right)$.

Tеорема 1 [6, с. 50]. Пусть $f \in L^{1}\left(\mathbb{R}^{n}\right)$. Если $\frac{\partial^{m} f}{\partial x_{k_{1}} \ldots \partial x_{k_{m}}} \in L^{p}\left(\mathbb{R}^{n}\right)$ nрu каком-нибудь $1<p \leqslant 2$ для всех $m=1,2, \ldots, n$ и любых $k_{1}, \ldots, k_{m}$, то $f(x) \in R^{0}\left(\mathbb{R}^{n}\right)$.

Tеорема 2 [4]. Пусть $\beta_{j}>0,1 \leqslant j \leqslant s, \beta_{0}=\min \left\{\beta_{1}, \ldots, \beta_{s}\right\}$. Имеют место следующие соотношения:

1) оператор $M_{\theta}^{\bar{\beta}}$ ограничен из $L^{p}$ в $L^{q}, 1<p \leqslant q<\infty$, тогда и только тогда, когда

$$
\frac{1}{p}+\frac{1}{q} \leqslant 1, \frac{1}{p}-\frac{n}{q} \leqslant \beta_{0} \quad \text { или } \frac{1}{p}+\frac{1}{q} \geqslant 1, \frac{n}{p}-\frac{1}{q} \leqslant \beta_{0}+(n-1) ;
$$

2) оператор $M_{\theta}^{\bar{\beta}}$ ограничен из $L^{1}$ в $L^{q}$ тогда и толъко тогда, когда

$$
1-\beta_{0}<\frac{1}{q} \leqslant 1
$$

3) оператор $M_{\theta}^{\bar{\beta}}$ ограничен из $H^{1}$ в $H^{1}$.

2. Основные результаты. Воспользуемся идеей обращения потенциалов с символами $\widehat{m^{\beta}}(\xi)=(2 \pi)^{n / 2} 2^{\beta-1}|\xi|^{1-\beta-n / 2} J_{\beta-1+n / 2}(|\xi|)$ из [7]. 
Обращение потенциала $f=M_{\theta}^{\bar{\beta}} \varphi, \varphi \in L^{p}, 1 \leqslant p \leqslant 2$, в неэллиптическом случае, когда $\operatorname{mes}\left\{\xi: \widehat{m_{\theta}^{\bar{\beta}}}(\xi)=0\right\}=0$, будем строить в виде

$$
T_{\theta}^{\bar{\beta}} f=\lim _{\varepsilon \rightarrow 0} \lim _{\delta \rightarrow 0} T_{\theta, \varepsilon, \delta}^{\beta} f,
$$

где

$$
\begin{gathered}
T_{\theta, \varepsilon, \delta}^{\bar{\beta}} f=F^{-1}\left(\widehat{\widehat{m_{\theta}^{\bar{\beta}}}(\xi)} e^{-\varepsilon|\xi|^{2}} /\left(\left|\widehat{m_{\theta}^{\bar{\beta}}}(\xi)\right|^{2}+i \delta\right)\right) * f \\
\widehat{m_{\theta}^{\bar{\beta}}}(\xi)=\int_{0}^{1} \rho^{n-1} \theta_{1}(\rho)\left(r_{1}^{2}-\rho^{2}+i 0\right)^{\beta_{1}-1} \times \ldots \\
\ldots \times \theta_{s-1}(\rho)\left(r_{s-1}^{2}-\rho^{2}+i 0\right)^{\beta_{s-1}-1} \theta_{s}(\rho)\left(1-\rho^{2}\right)_{+}^{\beta_{s}-1} d \rho \int_{S^{n-1}} e^{i(\rho \xi \cdot \sigma)} d \sigma .
\end{gathered}
$$

Следующая теорема даёт обращение потенциалов $M_{\theta}^{\bar{\beta}} \varphi, \varphi \in L^{p}, 1 \leqslant p \leqslant 2$.

Teоpema 3. Пусть $\varphi \in L^{p}, 1 \leqslant p \leqslant 2$. Тогда

$$
\left(T_{\theta}^{\bar{\beta}} M_{\theta}^{\bar{\beta}} \varphi\right)(x)=\varphi(x),
$$

где $T_{\theta}^{\bar{\beta}}$ - оператор (3).

Доказ ательств о. Из теоремы 1 следует, что оператор (4)-оператор свёртки с суммируемым ядром. Рассуждения будем основывать на равенстве

$$
\left(T_{\theta, \varepsilon, \delta}^{\bar{\beta}} M_{\theta}^{\bar{\beta}} \varphi\right)(x)=\left(W_{\varepsilon} \varphi\right)(x)-i \delta\left(N_{\theta, \varepsilon, \delta}^{\bar{\beta}} W_{\frac{\varepsilon}{2}} \varphi\right)(x), \quad \varepsilon, \delta>0,
$$

где

$$
N_{\theta, \varepsilon, \delta}^{\bar{\beta}} f=F^{-1}\left(n_{\theta, \varepsilon, \delta}^{\bar{\beta}}(\xi)\right) * f, \quad n_{\theta, \varepsilon, \delta}^{\bar{\beta}}(\xi)=e^{\frac{-\varepsilon|\xi|^{2}}{2}} /\left(\left|\widehat{m_{\theta}^{\bar{\beta}}}(\xi)\right|^{2}+i \delta\right) .
$$

Для функций $\varphi \in \Phi$ равенство (6) проверяется переходом к образам Фурье. Это равенство распространяется по ограниченности на все пространство $L^{p}$, $1<p \leqslant 2$, с учетом того, что операторы в обеих частях (6) ограничены из $L^{p}$ в $L_{\gamma}^{1}$, при некотором $\gamma>0$, где

$$
L_{\gamma}^{1}=\left\{f(x): \int_{\mathbb{R}^{n}} \frac{|f(x)| d x}{(1+|x|)^{\gamma}}<\infty\right\} .
$$

В случае $p=1$ равенство (6) выполняется в смысле $\Phi^{\prime}$ :

$$
\left\langle T_{\theta, \varepsilon, \delta}^{\bar{\beta}} M_{\theta}^{\bar{\beta}} \varphi, \omega\right\rangle=\left\langle W_{\varepsilon} \varphi-i \delta N_{\theta, \varepsilon, \delta}^{\bar{\beta}} W_{\frac{\varepsilon}{2}} \varphi, \omega\right\rangle, \quad \omega \in \Phi .
$$

Из (7) следует, что

$$
\left(T_{\theta, \varepsilon, \delta}^{\bar{\beta}} M_{\theta}^{\bar{\beta}} \varphi\right)(x)=\left(W_{\varepsilon} \varphi\right)(x)-i \delta\left(N_{\theta, \varepsilon, \delta}^{\bar{\beta}} W_{\frac{\varepsilon}{2}} \varphi\right)(x)+P(x),
$$


где $P(x)$ - некоторый многочлен. Так как функции $\left(T_{\theta, \varepsilon, \delta}^{\bar{\beta}} M_{\theta}^{\bar{\beta}} \varphi\right)(x),\left(W_{\varepsilon} \varphi\right)(x)$ и $\left(N_{\theta, \varepsilon, \delta}^{\bar{\beta}} W_{\frac{\varepsilon}{2}} \varphi\right)(x)$ принадлежат пространству $L^{1}, P(x) \equiv 0$ в $(8)$.

С учётом того, что $W_{\varepsilon} \varphi \rightarrow \varphi$ при $\varepsilon \rightarrow 0$ по $L^{p}$-норме или почти всюду, формула (5) будет следовать из равенства

$$
\lim _{\delta \rightarrow 0} \delta^{2}\left\|\left(N_{\theta, \varepsilon, \delta}^{\bar{\beta}} W_{\frac{\varepsilon}{2}} \varphi\right)(x)\right\|_{2}^{2}=0 .
$$

Докажем (9). Применяя равенство Парсеваля, получаем

$$
\delta^{2}\left\|\left(N_{\theta, \varepsilon, \delta}^{\bar{\beta}} W_{\frac{\varepsilon}{2}} \varphi\right)(x)\right\|_{2}^{2}=\frac{1}{(2 \pi)^{n}} \int_{\mathbb{R}^{n}} \frac{\delta^{2} e^{\frac{-\varepsilon|\xi|^{2}}{2}}}{\left|m_{\theta}^{\bar{\beta}}(\xi)\right|^{4}+\delta^{2}}\left|\left(\widehat{W_{\frac{\varepsilon}{2}} \varphi}\right)(\xi)\right|^{2} d \xi \rightarrow 0
$$

при $\delta \rightarrow 0$, с учётом того, что

$$
\lim _{\delta \rightarrow 0} \frac{\delta^{2} e^{\frac{-\varepsilon|\xi|^{2}}{2}}}{\left|m_{\theta}^{\bar{\beta}}(\xi)\right|^{4}+\delta^{2}}\left|\left(\widehat{W_{\frac{\varepsilon}{2}} \varphi}\right)(\xi)\right|^{2}=0, \quad \xi \notin\left\{\xi: \widehat{m_{\theta}^{\bar{\beta}}}(\xi)=0\right\} .
$$

Предельный переход (10) обосновывается применением мажорантной теоремы Лебега с учетом оценки

$$
\left(\delta^{2} e^{\frac{-\varepsilon|\xi|^{2}}{2}} /\left(\left|\widehat{m_{\theta}^{\bar{\beta}}}(\xi)\right|^{4}+\delta^{2}\right)\right)\left|\left(\widehat{W_{\frac{\varepsilon}{2}} \varphi}\right)(\xi)\right|^{2} \leqslant e^{\frac{-\varepsilon|\xi|^{2}}{2}}\left|\left(\widehat{W_{\frac{\varepsilon}{2}} \varphi}\right)(\xi)\right|^{2} \in L_{1} .
$$

Здесь существенным являлся тот факт, что $W_{\frac{\varepsilon}{2}} \varphi \in L^{2}$, если $\varphi \in L^{p}, 1 \leqslant p \leqslant 2$. Кроме того, учтено, что функция

$$
\widehat{\widehat{m_{\theta}^{\bar{\beta}}}(\xi)} e^{\frac{-\varepsilon|\xi|^{2}}{2}} /\left(\left|\widehat{m_{\theta}^{\bar{\beta}}}(\xi)\right|^{2}+i \delta\right)
$$

является 2-мультипликатором.

Описание образа $M_{\theta}^{\bar{\beta}}\left(L^{p}\right), 1 \leqslant p \leqslant 2$, даёт следующая теорема.

Tеорема 4. Пусть $\bar{\beta}=\left(\beta_{1}, \ldots, \beta_{s}\right), \beta_{j}>0,1 \leqslant j \leqslant s u 1 \leqslant p \leqslant 2$. Тогда

$$
M_{\theta}^{\bar{\beta}}\left(L^{p}\right)=\left\{f \in L^{q}: T_{\theta}^{\bar{\beta}} f \in L^{p}\right\},
$$

где $T_{\theta}^{\bar{\beta}}$ - оператор (3), q-произвольное число, $1 \leqslant q \leqslant 2$, такое, что оператор $M_{\theta}^{\bar{\beta}}$ ограничен из $L^{p}$ в $L^{q}$.

Доказательство. Вложение

$$
M_{\theta}^{\bar{\beta}}\left(L^{p}\right) \subset\left\{f \in L^{q}: T_{\theta}^{\bar{\beta}} f \in L^{p}\right\}
$$

вытекает из теоремы 2. 
Докажем вложение

$$
M_{\theta}^{\bar{\beta}}\left(L^{p}\right) \supset\left\{f \in L^{q}: T_{\theta}^{\bar{\beta}} f \in L^{p}\right\},
$$

обратное к (11). Пусть функция $\omega \in \mathcal{S}$ такова, что $\widehat{\omega}(\xi)=0$ в некоторой окрестности множества $V=\left\{\xi: \widehat{m_{\theta}^{\beta}}(|\xi|)=0\right\}$ (следовательно, $\omega \in \Phi_{V}$ ).

Имеем

$$
\left\langle M_{\theta}^{\bar{\beta}} T_{\theta}^{\bar{\beta}} f, \omega\right\rangle=\left\langle T_{\theta}^{\bar{\beta}} f, \overline{M_{\theta}^{\bar{\beta}}} \omega\right\rangle=\left\langle\lim _{\varepsilon \rightarrow 0} \lim _{\delta \rightarrow 0} T_{\theta, \varepsilon, \delta}^{\bar{\beta}} f, \overline{M_{\theta}^{\bar{\beta}}} \omega\right\rangle
$$

где $\overline{M_{\theta}^{\bar{\beta}}}$-оператор свёртки с символом $\overline{m_{\theta}^{\bar{\beta}}}(|\xi|)$. С учётом (12) и того факта, что сходимость по $L^{p}$ норме предполагает сходимость в $\Phi_{V}^{\prime}$, получаем

$$
\begin{array}{r}
\left\langle M_{\theta}^{\bar{\beta}} T_{\theta}^{\bar{\beta}} f, \omega\right\rangle=\lim _{\varepsilon \rightarrow 0}\left\langle\lim _{\delta \rightarrow 0} T_{\theta, \varepsilon, \delta}^{\bar{\beta}} f, \overline{M_{\theta}^{\bar{\beta}}} \omega\right\rangle=\lim _{\varepsilon \rightarrow 0} \lim _{\delta \rightarrow 0}\left\langle T_{\theta, \varepsilon, \delta}^{\bar{\beta}} f, \overline{M_{\theta}^{\bar{\beta}}} \omega\right\rangle= \\
=\lim _{\varepsilon \rightarrow 0} \lim _{\delta \rightarrow 0}\left\langle f, \overline{T_{\theta, \varepsilon, \delta}^{\bar{\beta}}} \overline{M_{\theta}^{\bar{\beta}}} \omega\right\rangle,
\end{array}
$$

где $\overline{T_{\theta, \varepsilon, \delta}^{\bar{\beta}}}-$ мультипликаторный оператор с символом

$$
\left(\widehat{m_{\theta}^{\bar{\beta}}}(\xi) e^{-\varepsilon|\xi|^{2}}\right) /\left(\left|\widehat{m_{\theta}^{\bar{\beta}}}(\xi)\right|^{2}-i \delta\right) \text {. }
$$

Далее имеем

$$
\left.\overline{\left(T_{\theta, \varepsilon, \delta}^{\bar{\beta}}\right.} \overline{M_{\theta}^{\bar{\beta}}} \omega\right)(x)=\left(W_{\varepsilon} \omega\right)(x)+i \delta\left(\overline{N_{\theta, \varepsilon, \delta}^{\bar{\beta}}} W_{\frac{\varepsilon}{2}} \omega\right)(x), \quad \varepsilon, \delta>0,
$$

где $\overline{N_{\theta, \varepsilon, \delta}^{\bar{\beta}}}-$ ограниченный в $L^{2}$ оператор, порождаемый 2-мультипликатором

$$
e^{\frac{-\varepsilon|\xi|^{2}}{2}} /\left(\left|\widehat{m_{\theta}^{\bar{\beta}}}(\xi)\right|^{2}-i \delta\right)
$$

Равенство (14) проверяется переходом к преобразованиям Фурье.

С учётом (13) и (14) получаем

$$
\left\langle M_{\theta}^{\bar{\beta}} T_{\theta}^{\bar{\beta}} f, \omega\right\rangle=\lim _{\varepsilon \rightarrow 0}\left\langle f, W_{\varepsilon} \omega\right\rangle+\lim _{\varepsilon \rightarrow 0} \lim _{\delta \rightarrow 0}\left\langle f, i \delta \overline{N_{\theta, \varepsilon, \delta}^{\bar{\beta}}} W_{\frac{\varepsilon}{2}} \omega\right\rangle .
$$

Докажем, что

$$
\lim _{\delta \rightarrow 0}\left\langle f, i \delta \overline{N_{\theta, \varepsilon, \delta}^{\bar{\beta}}} W_{\frac{\varepsilon}{2}} \omega\right\rangle=0 .
$$

Так как $\left.\overline{\left(N_{\theta, \varepsilon, \delta}^{\bar{\beta}}\right.} W_{\frac{\varepsilon}{2}} \omega\right)(x) \in \Phi_{V}$,

$$
\left\langle f, i \delta \overline{N_{\theta, \varepsilon, \delta}^{\bar{\beta}}} W_{\frac{\varepsilon}{2}} \omega\right\rangle=(2 \pi)^{-n}\left\langle\widehat{f}, i \delta F\left(\overline{N_{\theta, \varepsilon, \delta}^{\bar{\beta}}} W_{\frac{\varepsilon}{2}} \omega\right)\right\rangle,
$$


где преобразование Фурье $\widehat{f}$, понимаемое в смысле $\Phi^{\prime}$, совпадает с преобразованием Фурье в пространстве $L^{q^{\prime}}, \frac{1}{q}+\frac{1}{q^{\prime}}=1$ (в соответствии с теоремой Хауссдорфа-Юнга).

Применяя неравенство Гёльдера, будем иметь

$$
\left|\left\langle\widehat{f}, i \delta F \overline{\left(N_{\theta, \varepsilon, \delta}^{\bar{\beta}}\right.} W_{\frac{\varepsilon}{2}} \omega\right)\right\rangle \mid \leqslant \delta\|\widehat{f}\|_{q^{\prime}}\left\{\int_{\mathbb{R}^{n}} \frac{e^{-\varepsilon q|\xi|^{2}}|\widehat{\omega}(\xi)|^{q}}{\left|\widehat{m_{\theta}^{\bar{\beta}}}(\xi)\right|^{2 q}} d \xi\right\}^{\frac{1}{q}} .
$$

Заметим, что интеграл в правой части (17) конечен, так как $\widehat{\omega}(\xi)=0$ в некоторой окрестности множества $V$.

Переходя в $(17)$ к пределу при $\delta \rightarrow 0$, получаем (16).

Из (15) и (16) следует, что

$$
\left\langle M_{\theta}^{\bar{\beta}} T_{\theta}^{\bar{\beta}} f, \omega\right\rangle=\lim _{\varepsilon \rightarrow 0}\left\langle f, W_{\varepsilon} \omega\right\rangle=\langle f, \omega\rangle .
$$

Переходя к завершающему этапу доказательства, для заданной функции $\omega \in \mathcal{S}$ выберем последовательность $\left\{\omega_{N}\right\}, \omega_{N} \in \Phi_{V}$, такую, что $\widehat{\omega_{N}}(\xi)$ обращается в нуль в некоторой окрестности множества $V$ и

$$
\lim _{N \rightarrow \infty}^{\left(L^{q^{\prime}}\right)} \omega_{N}=\omega, \quad 1<q \leqslant 2 \quad \text { и } \quad \lim _{N \rightarrow \infty}^{\left(C_{0}\right)} \omega_{N}=\omega
$$

(возможность выбора такой последовательности доказана в [6, §3]).

Из (18) вытекает, что $\left\langle M_{\theta}^{\bar{\beta}} T_{\theta}^{\bar{\beta}} f, \omega_{N}\right\rangle=\left\langle f, \omega_{N}\right\rangle$. Переходя в этом равенстве к пределу при $N \rightarrow \infty$ на основании мажорантной теоремы Лебега, получаем $\langle f, \omega\rangle=\left\langle M_{\theta}^{\bar{\beta}} T_{\theta}^{\bar{\beta}} f, \omega\right\rangle, \omega \in \mathcal{S}$, откуда следует, что $f(x)=\left(M_{\theta}^{\bar{\beta}} T_{\theta}^{\bar{\beta}} f\right)(x)$ для почти всех $x \in \mathbb{R}^{n}$.

3. Обращение и описание потенциалов $\boldsymbol{M}_{\boldsymbol{\theta}}^{\bar{\beta}} \boldsymbol{\varphi}$ с $\boldsymbol{H}^{\mathbf{1}}$-плотностями. Обращение потенциала $f=M_{\theta}^{\bar{\beta}} \varphi, \varphi \in H^{1}$ в неэллиптическом случае будем строить в виде

$$
T_{\theta}^{\bar{\beta}} f=\lim _{\varepsilon \rightarrow 0}^{\left(H^{1}\right)\left(L^{2}\right)} \lim _{\delta \rightarrow 0}^{\bar{\beta}} T_{\theta, \varepsilon, \delta} f,
$$

где $T_{\theta, \varepsilon, \delta}^{\bar{\beta}}$ определяется равенством (4).

Теорема 5. Пусть $\varphi \in H^{1}$. Тогда

$$
M_{\theta}^{\bar{\beta}}\left(H^{1}\right)=\left\{f \in H^{1}: T_{\theta}^{\bar{\beta}} f \in H^{1}\right\}
$$

где $T_{\theta}^{\bar{\beta}}$ - оператор (19).

Доказательство теоремы 5 проводится по той же схеме, что и доказательство теорем 3 и 4.

\section{БИБЛИОГРАФИЧЕСКИЙ СПИСОК}

1. Strichartz R.S. Convolutions with kernels having singularities on a sphere// Trans. Amer. Math. Soc., 1970. Vol. 146. Pp. 461-471. 
2. Гиль А.В., Ногин В.А. Оценки для некоторых операторов типа потенциала с осциллирующими символами // Владикавк. матем. журн., 2010. Т. 12, №3. С. 2129. [Gil A.V., Nogin V.A. Estimates for some potential-type operators with oscillating symbols // Vladikavkaz. Mat. Zh., 2010. Vol.12, no. 3. Pp. 21-29].

3. Гиль А. В., Ногин В.А. $H^{p}-H^{q}$ оценки для некоторых операторов типа потенциала с осциллирующими символами // Изв. вузов. Сев.-Кав. регион, 2010. №5. С. 8-13. [Gil A. V., Nogin V.A. $H^{p}-H^{q}$ estimates for some potential-type operators with oscillating symbols // Izv. vuzov. Sev.-Kav. Region, 2010. no. 5. Pp. 8-13].

4. Гиль А. В., Задорожный А. И., Ногин В. А. Оценки для некоторых операторов свёртки с особенностями ядер на сферах // Вестн. Сам. гос. техн. ун-та. Сер. Физ.-мат. науки, 2011. № 2(23). C. 17-23. [Gil A. V., Zadorozhnyi A.I., Nogin V. A. Estimates for some convolution operators with singularities of their kernels on spheres // Vestn. Samar. Gos. Tekhn. Univ. Ser. Fiz.-Mat. Nauki, 2011. no. 2(23). Pp. 17-23].

5. Miyachi A. On some singular Fourier multipliers // J. Fac. Sci. Univ. Tokyo., Sect. 1 A, Math., 1981. Vol. 28, no. 2. Pp. 267-315.

6. Самко С.Г. Гиперсингулярные интегралы и их приложения. Ростов-на-Дону: Изд-во Ростов. ун-та, 1984. 208 с. [Samko S. G. Hypersingular integrals and their applications. Rostov-na-Donu: Izd-vo Rostov. Un-ta, 1984. 208 pp.]

7. Nogin V.A., Luzhetskaya P.A. Inversion and description of the ranges of multiplier operators of Strichartz-Peral-Miyachi-type // Fractional Calculus \&3 Applied Analysis, 2000. Vol. 3, no. 1. Pp. 87-96.

8. Stein E. M. Harmonic Analysis: Real-variable Method, Orthogonality, and Oscillatory Integrals. Princeton, NJ.: Princeton Univ. Press, 1993. 695 pp.

Поступила в редакцию 03/VIII/2011;

в окончательном варианте - 22/XI/2011.

\section{MSC: 45E10; 35L05}

\section{INVERSION AND CHARACTERIZATION OF SOME POTENTIALS WITH THE DENSITIES IN $L^{P}$ IN THE NON-ELLIPTIC CASE}

\section{A. V. Gil, A.I. Zadorozhnyi, V.A. Nogin}

Southern Federal University,

Faculty of Mathematics, Mechanics and Computer Science,

8a, Mil'chakova str., Rostov-on-Don, 344090, Russia.

E-mails: gil-alexey@yandey.ru, simon@rsu.ru, vnogin@math.rsu.ru

We construct the inversion of generalized Strichartz potentials with singularities of the kernels on a finite union of spheres in $\mathbb{R}^{n}$ with densities from space $L^{p}, 1 \leqslant p \leqslant 2$ and Hardy space $H^{1}$ in the non-elliptic case, when its symbols degenerate on a set of zero measure in $\mathbb{R}^{n}$. We also give the description of these potentials in terms of the inverting constructions.

Key words: convolution, oscillating symbol, multiplier, distribution.

Original article submitted 03/VIII/2011; revision submitted $22 / \mathrm{XI} / 2011$.

Alexey V. Gil (Ph. D. (Phys. \& Math.)), Senior Lecturer, Dept. of Differential and Integral Equations. Anatoliy I. Zadorozhnyi (Dr. Sci. (Phys. \& Math.)), Head of Dept., Dept. of Differential and Integral Equations. Vladimir A. Nogin (Ph.D. (Phys. \& Math.)), Associate Professor, Dept. of Differential and Integral Equations. 